\title{
PRICING FUTURES AND OPTIONS IN ELECTRICITY MARKETS
}

\author{
FRED ESPEN BENTH AND MAREN DIANE SCHMECK
}

\begin{abstract}
In this paper we derive power futures prices from a two-factor spot model being a generalization of the classical Schwartz-Smith commodity dynamics. We include non-Gaussian effects by introducing Lévy processes as the stochastic drivers, and estimate the model to data observed at the European Electricity Exchange in Germany. The spot and futures price models are fitted jointly, including the market price of risk parameterized from an Esscher transform. We apply this model to price call and put options on power futures. It is argued theoretically that the pricing measure for options may be different to the pricing measure of futures from spot in power markets due to the non-storability of the electricity spot. Empirical evidence pointing to this fact is found from option prices observed at the European Electricity Exchange.
\end{abstract}

\section{INTRODUCTION}

In the last two decades markets for power have been liberalized in Europe and other places world-wide. Nowadays, we find well-functioning markets for purchase of electricity in many countries on the European continent, in the Nordic countries and in the UK. Furthermore, there exists markets in North America, Australia and some places in Asia. Typically, these markets separate between a day-ahead spot market for electricity, and financial contracts for future delivery of power. In some, more developed markets, one also trades in derivatives like plain vanilla call and put options on the futures contracts. This takes place in for example the Nordic market NordPool and the German market European Electricity Exchange (EEX).

In this paper we focus the attention on pricing spot, futures and options jointly in the power market. Our aim is to argue for a separation of the modelling of the risk premium charged in the futures market and the risk neutral measure used for options pricing. The classical approach to futures pricing is to specify a stochastic dynamics of the spot price, and define the futures price as the conditional risk-adjusted expected average spot price over the delivery period. The risk-adjustment is modelled by a specification of pricing probabilities, which changes the characteristics of the spot dynamics (see Benth et al. [3] for a discussion and application of this approach to energy markets). Usually, as this approach yields a risk neutral (or martingale) dynamics of the futures price, one would price options using the same probability. We argue here that there is no violation of no-arbitrage pricing to have another pricing measure for options, as long as this is an equivalent martingale measure for the futures price dynamics. The economic argument in favour of this is the non-storability of the electricity spot price.

Based on a small data set of option prices at the EEX, we also argue empirically for this possibility. Fitting a two-factor model for the spot price dynamics to EEX data, we price futures and calibrate the risk premium using a parametric class of pricing probabilities stemming from the Esscher transform (see Benth et al. [3]). Although the access to option data at the EEX is poor due to a rather illiquid market, we find evidence for a different risk neutral pricing measure than the one used to derive futures prices from the spot dynamics. We benchmark our results to the Black-76 prices derived from historical volatility.

Our two-factor spot model is a generalization of the Schwartz-Smith dynamics (see Schwartz and Smith [15]), consisting of a long-term non-stationary factor and a short-term stationary factor. The SchwartzSmith model has been applied to electricity markets by Lucia and Schwartz [11], who analysed spot and futures data at the NordPool market. As the Schwartz-Smith model is Gaussian, it fails to account for the large spikes in the market. We extend the model to include Lévy process driven noises, which also accounts for the high variability in EEX prices in non-spike periods. Our proposed model is a simplification of the

Date: March 14, 2014.

Key words and phrases. Energy markets; Pricing measures; Jump processes; Spot price; Futures and forwards; Options.

Fred Espen Benth acknowledges the financial support from the project "Energy markets: modelling, optimization and simulation" (EMMOS), funded by the Norwegian Research Council under grant evita 205328/v30. 
dynamics proposed and analysed in Benth et al. [4] and [2]. The fitting of the spot and futures dynamics goes by filtering the non-stationary factor by using futures prices of contracts far from delivery.

The presentation of our results are separated into several sections. In the next section we present the rationale behind pricing of futures in power markets. Furthermore, we discuss the pricing of options, and why one may use a different probability for this purpose. Section 3 first defines the two-factor spot model, and presents theoretical futures prices based on this dynamics. The joint spot and futures price model is estimated to EEX data in section 4, while section 5 analyses empirically the option pricing performance of our futures price model. This section argues in favour of a different pricing measure for options. Finally, in section 6 , we conclude and outline some future research directions.

\section{THE RELATION BETWEEN SPOT, FUTURES AND OPTIONS IN POWER MARKETS}

Typically, the liberalized power markets are divided into a day-ahead spot market, a financial market for futures (and/or forwards ${ }^{1}$ ) contracts on power, and a market for plain vanilla call and put options on the futures. The futures contracts deliver the underlying power over an agreed period of time, and the delivery is settled financially, that is, the money-equivalent of the spot is delivered. These contracts are denominated in a "currency" per MWh and work essentially as a swap contract where one exchanges a floating spot price against a fixed over the contracted period.

For example, in the German EEX market the swaps have delivery periods being months, quarters or years. The swap price is naturally denominated in Euro per MWh, and the contract is accounted against the hourly power spot price. One distinguishes between base and peak load contracts, where the peak load take into account only the power spot prices in the peak hours, defined as the working days from 8 in the morning to 8 in the evening. The base load contracts are settled against the spot price of all hours in the delivery period.

The power spot prices are determined in an auction-based system, where the traders hand in prices and volumes for production or consumption for given hours the next day. Based on these bids, the exchange creates demand and supply curves for each hour the following day, and at 2pm the EEX publishes these spot prices for the 24 hours next day. We emphasise that the trade in the power spot market is physical, and one therefore needs to have facilities for either producing or consuming (retailing) electricity. Unlike most other assets that can be traded, one cannot form a portfolio and use the spot for investment or speculation purposes. By the very nature of electricity, it is not possible to store. There are some exceptions, since one may in fact use water reservoirs, say, as storage of power in terms of potential energy. However, this is only possible for a limited segment of the market, namely the hydro power producers.

The options traded in the market are written on specific financial swap contracts. At the EEX power options are written on the Phelix Base futures with monthly, quarterly and yearly delivery periods. The EEX offers only European style call and put options, where the exercise takes place four trading days prior to the beginning of the delivery period of the underlying futures.

Let us discuss at a more technical level the relationship between spot prices, swaps, and options. For illustration, consider first a market where the spot is a liquidly tradeable asset, like for example an exchangetraded stock. We denote $S(t)$ as the spot price at time $t \geq 0$, and consider a futures contract which delivers the spot at a maturity time $T$. The futures price at time $t \leq T$ is denoted by $f(t, T)$, and from standard no-arbitrage arguments based on the cash and carry strategy (see e.g. Duffie [10]), it can be determined as

$$
f(t, T)=S(t) \mathrm{e}^{r(T-t)} .
$$

Here, $r>0$ is the deterministic risk-free interest rate, where we have supposed that interest rates are continuously compounded. As is known from classical financial theory, (2.1) can be established without any model assumptions on the spot price.

Assume that we are given a complete filtered probability space $\left(\Omega, \mathcal{F},\left\{\mathcal{F}_{t}\right\}_{t \in[0, \widetilde{T}]}, P\right)$. We interpret $\widetilde{T}<\infty$ as the time horizon of the market, including the maturities of all options and futures relevant in our analysis. If $S(t)$ is a semimartingale process, then there exists (at least one) equivalent martingale measure $Q$ such that

$$
f(t, T)=\mathbb{E}_{Q}\left[S(T) \mid \mathcal{F}_{t}\right]
$$

\footnotetext{
${ }^{1}$ Some markets have both forwards and futures traded. We shall not make a distinction between these two asset classes here, but
} stick to the notion of futures. 
We refer to Shiryaev [14] for the rigorous argumentation with conditions leading to this representation of $f(t, T)$. In a complete market, that is, a market where all derivatives on $S$ can be replicated, the probability measure $Q$ is uniquely defined. In the case of an incomplete market, one may have many such measures $Q$. The question is to determine one relevant for pricing of derivatives. But, once such a measure is pinned down, we can price futures and next use the same probability for pricing options. Thus, for example the price of a European option with payoff $g(f(\tau, T))$ at exercise time $\tau \leq T$ becomes

$$
C(t)=\mathrm{e}^{-r(\tau-t)} \mathbb{E}_{Q}\left[g(f(\tau, T)) \mid \mathcal{F}_{t}\right],
$$

for $0 \leq t \leq \tau$. Note that we use the same $Q$ for both the futures and the option, as is the customary when pricing several derivatives based on an asset in an incomplete market situation. Note, however, that we may use different equivalent martingale measures for pricing different derivatives, as long as there exists at least one measure $Q$ that is an equivalent martingale measure for all products.

To see this, suppose that we have two derivatives on the spot with payoffs given by the random variables $X$ and $Y$, respectively. Let the prices at time zero be $C_{X}=\mathbb{E}_{Q_{X}}[X]$ and $C_{Y}=\mathbb{E}_{Q_{Y}}[Y]$, where we for the moment assume that the interest rate is zero to simplify the argument. The probabilities $Q_{X}$ and $Q_{Y}$ are equivalent martingale measures. If there exists an equivalent martingale measure $Q$, such that the price processes $S, C_{X}$ and $C_{Y}$, are all $Q$-martingales, then the market is arbitrage-free. However, as long as $Q$ is equivalent to $P$, it has to be equivalent to $Q_{X}$ and $Q_{Y}$ as well. Furthermore, by the no-arbitrage theory we must have that $C_{X}=\mathbb{E}_{Q}[X]$ and $C_{Y}=\mathbb{E}_{Q}[Y]$. This implies that

$$
\mathbb{E}_{Q_{X}}\left[X \frac{d Q}{d Q_{X}}\right]=\mathbb{E}_{Q_{X}}[X],
$$

and

$$
\mathbb{E}_{Q_{Y}}\left[Y \frac{d Q}{d Q_{Y}}\right]=\mathbb{E}_{Q_{Y}}[Y]
$$

These two equalities put strong conditions on the range of possible probabilities $Q_{X}, Q_{Y}$ and $Q$.

In the case of power markets, the situation is completely different since the probability measure used to price futures can theoretically be completely detached from the measure pricing options on futures. As we have already argued, the power spot price cannot be traded in the normal financial sense, and it works as a reference index for the settlement of futures contracts. With this view at hand, the pricing measure $Q$ used to derive the futures price on the spot does not need to be an equivalent martingale measure, but is required only to be an equivalent measure. However, the futures is a tradeable asset and its price dynamics must be a $Q$-martingale in order for the market to be free of arbitrage opportunities. Pricing using conditional expectation as in (2.2) ensures this by definition.

In a specification of the market, one would typically model the spot price evolution using some stochastic process $S(t)$, and choose a parametric class of equivalent probability measures $Q$. Based on a selected probability $Q$ from this class, the standard approach to price electricity futures is to define it as

$$
F\left(t, T_{1}, T_{2}\right)=\mathbb{E}_{Q}\left[\frac{1}{T_{2}-T_{1}} \int_{T_{1}}^{T_{2}} S(t) d t \mid \mathcal{F}_{t}\right] .
$$

Here, we consider a contract delivering electricity over the time interval $\left[T_{1}, T_{2}\right]$, and the contract is entered at time $t \leq T_{1}$, with settlement at the end of the delivery period $T_{2}$. Note that the price is denoted in $\mathrm{MWh}$, and therefore is normalized by the length of the delivery period. This gives a theoretical swap price dynamics which we next calibrate to the observed prices by fitting the parameters of the probabilities $Q$. This will pin down a probability $\widehat{Q}$ under which we model the risk-neutral futures price dynamics. Note that the risk-neutral dynamics of $F$ is a $\widehat{Q}$-martingale. Since by construction $\widehat{Q}$ is equivalent to $P$, we can also (in principle) derive the market dynamics of the futures. Note that in general $\widehat{Q}$ is not a probability for which the spot price dynamics becomes a martingale after discounting.

In reality, the above procedure in specifying a probability $\widehat{Q}$ for pricing futures is an approach to find a parametric representation of the price process $F\left(t, T_{1}, T_{2}\right)$, where we calibrate to represent the risk premium in the market, that is, to explain the difference between the observed futures prices and the predicted average spot price. The latter is calculated by relation (2.3) using $Q=P$. Apriori there are two extreme choices one can make on $Q$. First, ignoring the existence of a risk premium, one could select $Q=P$. Alternatively, assuming the electricity spot is tradeable, one could force $Q$ to be a martingale measure. 
Note that depending on the model for $S$, one could have many possible martingale measures, so the latter choice is not necessarily unique. Both alternatives are theoretically viable, but hardly reasonable from the characteristics of electricity markets.

Our next problem is to price call and put options written on the futures. Following the standard noarbitrage pricing framework discussed above, a first thought would be to use $\widehat{Q}$ and compute the option price using this probability. To be more specific, let us suppose that we have a call option with exercise time $\tau \leq T_{1}$ written on a swap with dynamics $F\left(t, T_{1}, T_{2}\right)$ given in (2.3) for the pricing measure $\widehat{Q}$. The price of this call at time $t \leq \tau$ is

$$
C(t)=\mathrm{e}^{-r(\tau-t)} \mathbb{E}_{\widehat{Q}}\left[\max \left(F\left(\tau, T_{1}, T_{2}\right)-K, 0\right) \mid \mathcal{F}_{t}\right] .
$$

However, in general, there will exist several equivalent measures $Q$ for which $t \mapsto F\left(t, T_{1}, T_{2}\right)$ is a $Q$ martingale. In fact, since typically the power spot price dynamics involves jump processes, the futures price will follow a jump dynamics as well. Under certain conditions, such models admit the existence of a continuum of equivalent martingale measures $Q$. In this case we pin down a pricing measure $\widetilde{Q}$ by selecting it from a parametric class of equivalent martingale measures $Q$ for $F\left(t, T_{1}, T_{2}\right)$. One could derive this probability by calibrating to observed option prices in the market, or to appeal (partial) hedging arguments (see Cont and Tankov [9] for a discussion of hedging and pricing in incomplete markets).

Note that finding $\widetilde{Q}$ for option pricing follows in principle the same scheme as choosing $\widehat{Q}$ for the futures prices. The fundamental difference is that $\widehat{Q}$ does not need to be a martingale measure for the spot price, whereas $\widetilde{Q}$ has to be a martingale measure for the futures price. Both probability measures are equivalent to $P$. In the next sections we shall estimate a particular two-factor model to spot price data collected from the EEX, and apply this to futures pricing based on a class of probabilities defined by Esscher transformation. Using option price data, we shall argue that the spot-futures probability $\widehat{Q}$ is not the right probability for pricing options on the futures, pointing towards $\widetilde{Q} \neq \widehat{Q}$.

Our analysis is not restricted to power markets only. In the weather markets, like the temperature market at the Chicago Mercantile Exchange (CME), futures on temperature indices measured in various cities world-wide are traded. In addition, plain vanilla call and put options on these futures are traded. The underlying "spot" price here is the temperature in a given city, for example Chicago itself. Given a stochastic model for the temperature $S(t)$, one can derive the resulting futures price written on an index of the temperature. Typically, one chooses to price using a conditional expectation analogous to (2.3), where a pricing measure is selected. Obviously, temperature itself is not a tradeable commodity, and we can use the same argumentation as above to defend choosing the pricing probabilities which are not necessarily martingale measures for the temperature dynamics. On the other hand, the futures contracts are tradeable financial assets, and to price the options with these as underlying, we need to use a probability measure $Q$ which turns the futures price into a $Q$-martingale. As in the case of power, the futures pricing measure $\widehat{Q}$ does not need to be the same as the option pricing measure $\widetilde{Q}$. We note in passing that CME also organize a market for precipitation derivatives based on snow and rainfall indices in some cities in the US. Further, there has been trials to create an organized market for wind futures and options at the now closed US Futures Exchange. Here our discussion makes sense as well.

\section{THE SPOT PRICE DYNAMICS AND IMPLIED FUTURES PRICES}

We consider a simple arithmetic two-factor spot price dynamics in the spirit of Lucia and Schwartz [11]. The occurrence of negative spikes at the EEX, and, even more, the observation that these spikes may even lead to negative prices, indicate that an arithmetic model may be suitable. To this end, suppose that $S(t)$ follows the dynamics

$$
S(t)=\Lambda(t)+X(t)+Y(t) .
$$

Here, $\Lambda:[0, \widetilde{T}] \mapsto \mathbb{R}$ is a measurable deterministic function, modelling the mean seasonal variation in spot prices. Usually, this function consists of a linear trend and a periodic function (a linear combination of sines and cosines, with different frequencies), and as such is a smooth function. The base component $X(t)$ in the spot price dynamics is assumed to be non-stationary and defined to be a Lévy process, i.e.,

$$
d X(t)=d L_{1}(t) .
$$


In Lucia and Schwartz [11], it is assumed that $L_{1}(t)=\gamma t+\sigma B(t)$ with $\gamma$ and $\sigma$ being constants and $B(t)$ a Brownian motion. The volatility $\sigma$ is naturally assumed to be positive. One may think of the base component as stochastic variations from market activity as well as long term effects like inflation in fuel prices and limited resources, as well as entry of new sources of energy (like renewables). As it will turn out from our empirical analysis of EEX spot price data, a drifted Brownian motion is unsuitable for modelling the true dynamics of the non-stationary term, and a Lévy process is much more appropriate.

Typically in power markets spot prices may exhibit random shocks due to imbalances in supply and demand. These shocks are seen as spikes in the price path, imposed from an unexpected increase in demand due to colder weather, say, or shut down of a major power plant yielding a drop in supply. The prices will in both these cases exhibit a major price jump upward, which is followed typically by a strong decline since demand will be significantly reduced by higher prices, or expensive power production plants are ramped up (like coal-fired plants in Denmark in the NordPool area). In the EEX market one observes many negative spikes, which is caused by wind power mainly. By political legislation, wind power and other renewable energy sources have priority into the electricity grid, and hence an unexpected increase in wind power production (due to more wind where the farms are...) may create bigger than expected supply (since it takes time to ramp down or adjust other power plants fueled by gas and coal or producing nuclear energy). In fact, one observes negative prices in the EEX market due to over-supply, where some producers choose to pay for power consumption rather than shut down their production.

From this discussion, we see that there is ample evidence for a mean-reverting short-time factor of the form

$$
d Y(t)=-\eta Y(t) d t+d L_{2}(t) .
$$

Here, the constant $\eta>0$ is expected to be rather big, since spikes created by the Lévy process $L_{2}(t)$ are reverting fastly back to normal price levels. We suppose that $L_{2}(t)$ may have both positive and negative jumps, that is, $L_{2}(1)$ is distributed on $\mathbb{R}$.

Notice that in Lucia and Schwartz [11], both an arithmetic and geometric two-factor model were analysed theoretically and empirically on NordPool data. In their approach, the second factor $Y$ was also assumed to be driven by a Brownian motion. We believe that a jump factor for the noise is more appropriate in order to explain the sudden spikes in prices, exhibiting a jump like behaviour in the price path. Also, most empirical studies of power spot prices point strongly towards non-Gaussianity in prices, and hence the need to use other processes than the Brownian motion to drive the dynamics (see discussion in Benth et al. [3]). We remark that Lucia and Schwartz [11] let the short and long term factors correlate through their driving noise.

We denote $L=\left(L_{1}, L_{2}\right)$, and assume that $L$ is a bivariate Lévy process with cumulant (log-characteristic function) defined by

$$
\psi(\mathbf{x})=\mathrm{i} \mu^{\prime} \mathbf{x}-\frac{1}{2} \mathbf{x}^{\prime} C \mathbf{x}+\int_{\mathbb{R}^{2}} \mathrm{e}^{\mathrm{i} \mathbf{x}^{\prime} \mathbf{z}}-1-\mathrm{i} \mathbf{x}^{\prime} \mathbf{z} 1(|\mathbf{z}| \leq 1) \ell(d \mathbf{z}),
$$

with $\mathbf{x}=(x, y)^{\prime} \in \mathbb{R}^{2}, \mu \in \mathbb{R}^{2}, C$ a symmetric non-negative definite $2 \times 2$ matrix and $\ell(d \mathbf{z})$ a Lévy measure on $\mathbb{R}^{2} \backslash\{\mathbf{0}\}$. Here $\mathrm{x}^{\prime}$ denotes the transpose of the vector, and $i=\sqrt{-1}$ is the imaginary unit. In the case of independence between $L_{1}$ and $L_{2}$, we can express the cumulant as a sum

$$
\psi(x, y)=\psi_{1}(x)+\psi_{2}(y)
$$

where $\psi_{i}, i=1,2$ are cumulants of the univariate Lévy processes $L_{1}$ and $L_{2}$. Our general model allows for a dependency between $L_{1}$ and $L_{2}$, although we shall assume independence in the empirical study on EEX data below.

In Benth et al. [4] they use a more general model. The stationary short time variations are modelled as a continuous-time autoregressive moving average (CARMA) process, where the driving process $L_{2}$ is an $\alpha$-stable Lévy process. As it includes mean reversion, a CARMA model is comparable to the standard approach of commodity spot price modelling, that is, to describe the spot as a sum of several OrnsteinUhlenbeck processes with different speeds of mean reversion and stochastic drivers (see Benth et al. [3]). In Benth et al. [4], a CARMA(2,1) dynamics is proposed and fitted empirically to EEX spot price data. Such a dynamics is similar to a two-factor model, with each factor being an Ornstein-Uhlenbeck process. Although we find strong indications of a two-factor dynamics in our empirical study, we simplify the 
considerations here to a one-factor model as a first order approximation of the short-term factor. This makes the fitting of data significantly easier, and is in line with the more classical two-factor model of Lucia and Schwartz [11]. Moreover, it turns out that we can do well with a much more regular Lévy process than the $\alpha$-stable to model the random fluctuations.

Our first concern is to introduce a parametric class of equivalent probabilities $Q$ which is appropriate for pricing swaps. For $\theta=\left(\theta_{1}, \theta_{2}\right) \in \mathbb{R}^{2}$, define the equivalent probability $Q_{\theta}$, where the density process of $Q_{\theta}$ with respect to $P$ is

$$
\left.\frac{d Q_{\theta}}{d P}\right|_{\mathcal{F}_{t}}=\exp \{\theta L(t)-\psi(-\mathrm{i} \theta) t\}
$$

In order for this to be well-defined, we must of course assume exponential integrability conditions on $L(1)$. Hence, suppose from now on that there exists a constant $c>0$ such that

$$
\int_{\mathbb{R}^{2}} \mathrm{e}^{\mathbf{x}^{\prime} \mathbf{z}} \ell(d \mathbf{z})<\infty
$$

for all $|\mathbf{x}| \leq c$. This ensures finite exponential moments for $L(1)$ up to order $c$.

The probability $Q_{\theta}$ parameterized by $\theta$ is known as the Esscher transform of $L$ (see Benth $e t$ al. [3]). The probability $Q_{\theta}$ is equivalent to $P$ by definition of the Radon-Nikodym densities. We emphasize, however, that we do not demand $Q_{\theta}$ to be a martingale measure, in the sense that the power spot dynamics becomes a $Q_{\theta}$-martingale (the reader should note that this is technically impossible anyway with the Esscher transform on an Ornstein-Uhlenbeck process, see Benth and Sgarra [6]). The reason is the non-storability of the spot which makes it non-tradeable, that is, one cannot create portfolios with spot investments in electricity. Once purchased, it must be consumed. The parameter $\theta$ is restricted to the subspace of $\mathbb{R}^{2}$ defined by $|\theta| \leq c$.

In the next Lemma we characterize the process $L$ under $Q_{\theta}$ :

Lemma 3.1. The process $L$ is a Lévy process with respect to $Q_{s}$ with cumulant function

$$
\psi_{Q_{\theta}}(\mathbf{x})=\psi(\mathbf{x}-\mathrm{i} \theta)-\psi(-\mathrm{i} \theta) .
$$

Hence, the drift is

and the Lévy measure

$$
\mu+\theta^{\prime} C+\int_{|\mathbf{z}|<1}\left(e^{\theta \mathbf{z}}-1\right) \mathbf{z} \ell(d \mathbf{z})
$$

$$
\ell_{Q_{\theta}}(d \mathbf{z})=\mathrm{e}^{\theta \mathbf{z}} \ell(d \mathbf{z})
$$

while the covariance matrix $C$ remains the same.

Proof. Using Bayes' Theorem along with the density process of $Q_{\theta}$ and the independent increment property of the Lévy process, yield that the conditional log-characteristic function of $L(t)$ given $\mathcal{F}_{s}$ for $t \geq s \geq$ 0 is

$$
\ln \mathbb{E}_{Q_{s}}\left[\mathrm{e}^{\mathrm{ix} \mathbf{x}^{\prime} L(t)} \mid \mathcal{F}_{s}\right]=(\psi(\mathbf{x}-\mathrm{i} \theta)-\psi(-\mathrm{i} \theta))(t-s) .
$$

Hence, $L$ is a Lévy process under $Q_{\theta}$ as well. By a direct computation, we find the drift and the Lévy measure as claimed.

Note that if we have a (bivariate) drifted Brownian motion as Lévy process, that is, $\ell(d \mathbf{z})=0$, then the Esscher transform is simply a Girsanov transform of the Brownian motion with a constant parameter $\theta$. For Lévy processes with jumps, the Lévy measure is exponentially tilted by the Esscher transform. We may interpret this as a rescaling of the size and intensity of jumps.

We remark that the expected value of $L(1)$ under $Q_{\theta}$ is given by

$$
\mathbb{E}_{\theta}[L(1)]=-\mathrm{i} \nabla \psi(-\mathrm{i} \theta),
$$

where $\nabla$ is the gradient and $\mathbb{E}_{\theta}[\cdot]$ is the expectation operator with respect to the probability $Q_{\theta}$. Thus, the Lévy process $\widetilde{L}(t)=L(t)+\mathrm{i} \nabla \psi(-\mathrm{i} \theta) t$ becomes a martingale under $Q_{\theta}$ as it has expectation zero. This means in particular that under $Q_{\theta}$, the dynamics of $X$ and $Y$ are, respectively,

$$
d X(t)=-\mathrm{i} \psi_{x}(-\mathrm{i} \theta) d t+d \widetilde{L}_{1}(t)
$$


and

$$
d Y(t)=\left\{-\mathrm{i} \psi_{y}(-\mathrm{i} \theta)-\eta Y(t)\right\} d t+d \widetilde{L}_{2}(t) .
$$

Here, we have used the notation $\psi_{x}$ and $\psi_{y}$ as the partial derivatives of $\psi$ with respect to the two variables $x$ and $y$, respectively. The solution $Y(s)$ at time $s \geq t$, conditioned on $Y(t)$, of this Ornstein-Uhlenbeck process is

$$
Y(s)=Y(t) \mathrm{e}^{-\eta(s-t)}+\frac{-\mathrm{i} \psi_{y}(-\mathrm{i} \theta)}{\eta}\left(1-e^{-\eta(s-t)}\right)+\int_{t}^{s} \mathrm{e}^{-\eta(u-t)} \widetilde{L}_{2}(d u) .
$$

Next, we consider pricing of swaps in this market. Let us start with analysing the implied swap price dynamics for the arithmetic model. The following result holds:

Proposition 3.2. The swap price $F\left(t, T_{1}, T_{2}\right)$ is given by

$$
\begin{aligned}
F\left(t, T_{1}, T_{2}\right)=\bar{\Lambda} & \left(T_{1}, T_{2}\right)+X(t)+Y(t) \bar{\eta}\left(t, T_{1}, T_{2}\right) \\
& -\frac{1}{2} \mathrm{i} \psi_{x}(-\mathrm{i} \theta)\left(T_{2}-T_{1}\right)-\mathrm{i} \psi_{x}(-\mathrm{i} \theta)\left(T_{1}-t\right)+\frac{-\mathrm{i} \psi_{y}(-\mathrm{i} \theta)}{\eta}\left(1-\bar{\eta}\left(t, T_{1}, T_{2}\right)\right),
\end{aligned}
$$

where

$$
\bar{\eta}\left(t, T_{1}, T_{2}\right)=\frac{1}{T_{2}-T_{1}} \int_{T_{1}}^{T_{2}} \mathrm{e}^{-\eta(s-t)} d s
$$

and $\bar{\Lambda}\left(T_{1}, T_{2}\right)$ is the average value of the seasonality function $\Lambda(s)$ over the interval $\left[T_{1}, T_{2}\right]$.

Proof. From the expression in (3.7), we find (for $s \geq t$ )

$$
\mathbb{E}_{Q_{\theta}}\left[X(s) \mid \mathcal{F}_{t}\right]=X(t)-\mathrm{i} \psi_{x}(-\mathrm{i} \theta)(s-t),
$$

after appealing to the independent increment property of the $Q_{\theta}$-Lévy process $\widetilde{L}_{1}$ with zero mean, and the $\mathcal{F}_{t}$-measurability of $X(t)$. Similarly, from the independent increment property of the $Q_{\theta}$-Lévy process $\widetilde{L}_{2}$, having mean zero, we find from (3.9)

$$
\mathbb{E}_{Q_{\theta}}\left[Y(s) \mid \mathcal{F}_{t}\right]=Y(t) \mathrm{e}^{-\eta(s-t)}-\frac{\mathrm{i} \psi_{y}(-\mathrm{i} \theta)}{\eta}\left(1-\mathrm{e}^{-\eta(s-t)}\right) .
$$

Since

$$
F\left(t, T_{1}, T_{2}\right)=\frac{1}{T_{2}-T_{1}} \int_{T_{1}}^{T_{2}}\left\{\Lambda(s)+\mathbb{E}_{Q_{\theta}}\left[X(s)+Y(s) \mid \mathcal{F}_{t}\right]\right\} d s
$$

the result follows after using the Fubini Theorem.

We note that $\bar{\eta}$ is the average value of the "volatility function" $\exp (-\eta(s-t))$ over the delivery period $\left[T_{1}, T_{2}\right]$, and takes the form

$$
\bar{\eta}\left(t, T_{1}, T_{2}\right)=\frac{1}{\eta\left(T_{2}-T_{1}\right)}\left(\mathrm{e}^{-\eta\left(T_{1}-t\right)}-\mathrm{e}^{-\eta\left(T_{2}-t\right)}\right)
$$

or,

$$
\bar{\eta}\left(t, T_{1}, T_{2}\right)=\mathrm{e}^{-\eta\left(T_{1}-t\right)} \frac{1}{\eta\left(T_{2}-T_{1}\right)}\left(1-\mathrm{e}^{-\eta\left(T_{2}-T_{1}\right)}\right) .
$$

In the representation (3.11), $T_{1}-t$ is time left until start of delivery, and $T_{2}-T_{1}$ is length of delivery. We recognize the exponential damping factor $\exp \left(-\eta\left(T_{1}-t\right)\right)$ as the Samuelson effect on the volatility, that is, the volatility of the spot is increasing as time to start of delivery is decreasing. The classical Samuelson effect says that the volatility of the futures price is exponentially increasing in time to maturity to the spot volatility (see Samuelson [13] and Benth et al. [3]). We note here that $\bar{\eta}\left(t, T_{1}, T_{2}\right)$ is not converging to the "spot volatility", being one in this context, but to a value less than this. The delivery period creates this violation of the classical Samuleson effect. It is natural from a financial and empirical point of view that the volatility of the electricity futures price is not converging to that of the spot as the futures price is the average of the spot over a delivery period.

We derive the dynamics of $F$ in the next proposition 
Proposition 3.3. The $Q_{\theta}$ dynamics of the swap price is

$$
d F\left(t, T_{1}, T_{2}\right)=d \widetilde{L}_{1}(t)+\bar{\eta}\left(t, T_{1}, T_{2}\right) d \widetilde{L}_{2}(t) .
$$

Proof. Since $\bar{\eta}^{\prime}\left(t, T_{1}, T_{2}\right)=\eta \bar{\eta}\left(t, T_{1}, T_{2}\right)$, the result follows after applying the Itô formula for jump processes and the $Q_{\theta}$-dynamics of $X$ and $Y$.

As is apparent from the definition of $F\left(t, T_{1}, T_{2}\right)$, it is a $Q_{\theta}$-martingale process for $t \leq T_{1}$. Thus, it defines an arbitrage-free model for the stochastic evolution of electricity futures prices.

\section{AN EMPIRICAL STUDY OF EEX SPOT AND FUTURES PRICES}

In this section we want to estimate the parameters in the spot model, and calibrate it to futures prices where we derive the market price of risk $\theta$. It turns out that a joint estimation of spot and futures is most efficient, where one can make use of the asymptotic behaviour of futures prices to filter out the nonstationary factor in the spot. This approach is analogous of the calibration procedure in Schwartz and Smith [15], with a more sophisticated version of it found in Benth et al. [4].

The following asymptotic result of the futures price with respect to time to delivery plays a crucial role in the estimation algorithm.

Proposition 4.1. It holds that

$$
\lim _{T_{1}-t \rightarrow \infty}\left\{F\left(t, T_{1}, T_{2}\right)-\bar{\Lambda}\left(T_{1}, T_{2}\right)-\Psi\left(t, T_{1}, T_{2} ; \theta\right)-X(t)\right\}=0
$$

where

$$
\Psi\left(t, T_{1}, T_{2} ; \theta\right)=-\frac{1}{2} \mathrm{i} \psi_{x}(-\mathrm{i} \theta)\left(T_{2}-T_{1}\right)-\mathrm{i} \psi_{x}(-\mathrm{i} \theta)\left(T_{1}-t\right)-\frac{\mathrm{i} \psi_{y}(-\mathrm{i} \theta)}{\eta}
$$

Proof. Recalling the explicit dynamics of $F\left(t, T_{1}, T_{2}\right)$ in Proposition 3.2, the result follows after observing that $\exp \left(-\eta\left(T_{1}-t\right)\right) \rightarrow 0$ as $T_{1}-t \rightarrow \infty$.

Hence, asymptotically the futures price behaves like

$$
F\left(t, T_{1}, T_{2}\right) \approx \bar{\Lambda}\left(T_{1}, T_{2}\right)+\Psi\left(t, T_{1}, T_{2} ; \theta\right)+X(t),
$$

for $T_{1}-t \rightarrow \infty$. This means that in the long end of the futures market, the prices fluctuate as the nonstationary factor $X(t)$ plus some non-stochastic adjustment term $\bar{\Lambda}\left(T_{1}, T_{2}\right)+\Psi\left(t, T_{1}, T_{2} ; \theta\right)$ involving the market price of risk $\theta$. From these considerations we can derive an algorithm for estimating the model. It goes as follows.

For a fixed delivery period $\left[T_{1}, T_{2}\right]$,

(1) Fit a seasonal function $\Lambda(t)$ to the spot prices $S(t)$.

(2) Fit the autocorrelation function of $Y(t)$ to the deseasonalized spot prices to have an apriori estimate of $\eta$. Use this $\eta$ to find a threshold $\widehat{T}$ for which " $T_{1}-t=\infty$ ", that is, how big should $T_{1}-t$ be for the asymptotic behaviour of $F$ in (4.1) to be acceptable.

(3) Subtract $\bar{\Lambda}\left(T_{1}, T_{2}\right)$ from the observed futures prices to "deseasonalize" them. Call this time series $\widetilde{F}\left(t, T_{1}, T_{2}\right)$.

(4) Observe that we have for $T_{1}-t \geq \widehat{T}$

$$
\widetilde{F}\left(t, T_{1}, T_{2}\right) \approx c\left(\theta, T_{1}, T_{2}\right)-\mathrm{i} \psi_{x}(-\mathrm{i} \theta)\left(T_{1}-t\right)+X(t),
$$

where

$$
c\left(\theta, T_{1}, T_{2}\right)=-\frac{1}{2} \mathrm{i} \psi_{x}(-\mathrm{i} \theta)\left(T_{2}-T_{1}\right)-\frac{\mathrm{i} \psi_{y}(-\mathrm{i} \theta)}{\eta} .
$$

Hence, for all observed futures prices $F\left(t, T_{1}, T_{2}\right)$ for which $T_{1}-t \geq \widehat{T}$, estimate the "constants" $c\left(\theta, T_{1}, T_{2}\right)$ and $-\mathrm{i} \psi_{x}(-\mathrm{i} \theta)$ by linear regression of $\widetilde{F}$ with respect to $T_{1}-t$.

(5) Using the estimated regression coefficients $\widehat{c}$ and $\widehat{a}$, we filter out $X(t)$ from the observations,

for all $T_{1}-t \geq \widehat{T}$.

$$
\widetilde{F}\left(t, T_{1}, T_{2}\right)-\widehat{c}-\widehat{a}\left(T_{1}-t\right)
$$




\begin{tabular}{cccccccc}
\hline$\xi_{0}$ & $\xi_{1}$ & $\xi_{2}$ & $\xi_{3}$ & $\xi_{4}$ & $\xi_{5}$ & $\tau_{1}$ & $\tau_{2}$ \\
\hline 738.733 & 4.360 & -11.716 & 0.020 & 1.000 & 1.000 & -13637.760 & 40.401 \\
\hline
\end{tabular}

TABLE 1. Estimated parameters of the seasonal function.

(6) Subtract the filtered data series $X(t)$ from the deseasonalized spot prices. This results in a time series which is modelled by $Y(t)$. Re-estimate $\eta$ based on linear regression of $Y(t)$ against $Y(t-$ 1).

(7) Fit a Lévy process $L$ to the residuals of the $Y$ process and the time series of the $X$ process obtained above. From the fitted Lévy process $L$, we obtain the cumulant $\psi$.

(8) For the given cumulant $\psi$, find the estimated market price of risk $\theta$ by solving the system of equations

$$
\begin{aligned}
& \widehat{a}=-\mathrm{i} \psi_{x}(-\mathrm{i} \theta), \\
& \widehat{c}=-\frac{1}{2} \mathrm{i} \psi_{x}(-\mathrm{i} \theta)\left(T_{2}-T_{1}\right)+\frac{-\mathrm{i} \psi_{y}(-\mathrm{i} \theta)}{\eta} .
\end{aligned}
$$

This calibration algorithm provides us with a full specification of both the spot and the futures price model, including the estimation of the market prices of risk $\theta=\left(\theta_{1}, \theta_{2}\right)$. We next apply it to spot and futures price data collected from the European Energy Exchange (EEX).

We have available daily Phelix base load spot prices from 02.01.2006 until 19.10.2008, constituting altogether 1022 daily observations. We remark that we include weekend prices as we are going to apply base load futures prices in our estimation routine. These futures are settled on the spot prices including the weekends. To the spot price data, we fit the seasonality function taken from Barndorff-Nielsen et al. [2],

$$
\Lambda(t)=\xi_{0}+\xi_{1} \cos \left(\frac{\tau_{1}+2 \pi t}{365}\right)+\xi_{2} \cos \left(\frac{\tau_{2}+2 \pi t}{7}\right)+\xi_{3} t+\xi_{4} \mathbf{1}_{\text {Sat }}(t)+\xi_{5} \mathbf{1}_{\text {Sun }}(t) .
$$

This function takes annual and weekly seasonality into account along with a trend. As prices on weekends are in general lower than during the rest of the week due to a different demand situation, we introduce additionally a weekend-correction to capture these effects. Here $\mathbf{1}_{\text {Sat }}(t)$ and $\mathbf{1}_{\text {Sun }}(t)$ are equal 1 , if the weekday corresponding to $t$ is a Saturday and Sunday, respectively.

A non-linear least squares estimation on the spot data yields the parameters reported in Table 1. Fig. 1 (left) displays the spot price data and its estimated seasonality function. The estimated seasonality follows the general movements of the spot, on a weekly pattern as well as a yearly one.

Next we continue the calibration algorithm with filtering the non-stationary factor $X$ from the futures data with long time to delivery. For this purpose we use base load futures contracts with 1 month delivery period from the EEX, for which we have available price data for the same dates as the spot (weekends and holidays are excluded, as there is no trade in futures).

We first need to determine the threshold $\widehat{T}$ for which the futures prices are asymptotically given by (4.1). This depends, obviously, on the value of $\eta$, the speed of mean reversion in the factor process $Y$. We can estimate this parameter from the autocorrelation function of $Y$ which is known to be exponentially decaying at the rate $\eta$ (see Benth et al. [3]). However, at this point in the estimation procedure we have not yet filtered the time series of $Y$ from the spot data, so the empirical autocorrelation function is unknown to us. Therefore, we do a rough estimation of $\eta$ by looking at the empirical autocorrelation of the deseasonalized spot, which is modelled by $X(t)+Y(t)$. We observe a decaying autocorrelation structure, and fit an exponentially decaying function to the first five lags obtaining the pre-estimate $\widehat{\eta}=0.1781$. We derive $\widehat{T}=16$ as the threshold when $Y(t) \bar{\eta}\left(t, T_{1}, T_{2}\right) \approx 1$ using $Y(t)$ being three times the standard deviation of spot price data. Note that we expect the presence of $X$ to make the beta smaller than the "true" one. A larger value for $\eta$ would lead to a smaller threshold. Hence, our decision to apply $\widehat{T}=16$ is a conservative choice.

We construct a time series of futures prices with "infinite" time to delivery from the base load contracts as follows: if the time to delivery is more than 16 days, we choose the futures which has the first coming month as delivery period. Otherwise, we switch to the contract with delivery in the following month. That is, we use the price series of front-month contracts as long as these are farther than 16 days to delivery, 

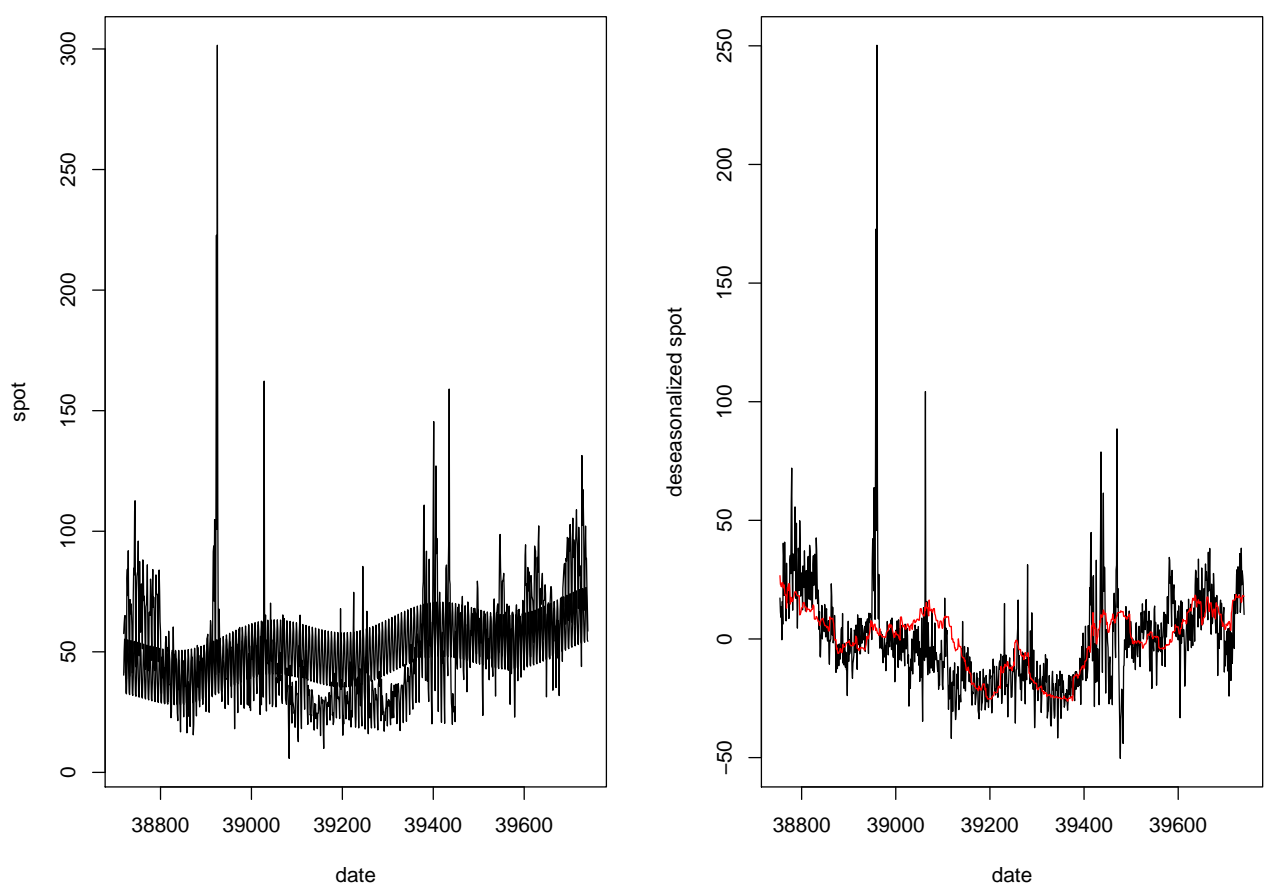

FIGURE 1. Left: empirical spot price data together with the estimated seasonality function. Right: deseasonalized spot price data with the filtered data series $X(t)$.

and switch to the next month when the front-month contracts have less than 16 days to delivery. Like this we make sure that for each date we have a futures price with time to delivery of more than 16 days. These prices will not, at least approximately, have any influence from the stationary component $Y$. As the futures are not traded on weekends and holidays, we use as a substitute for missing values at the weekend the price of the preceeding Friday. On holidays, we use the price of the last trading day before the holidays.

To deseasonalize the constructed futures price series we subtract the average seasonality of the delivery period. We have fitted the seasonality function to data until October 2008, such that we take October 2008 as the last delivery period and let our futures price series end at 14.09.2008. A linear regression of this time series delivers the estimates $\hat{a}=0.030$ and $\hat{c}=3.406$. We filter the non-stationary time series $X(t)$ from the futures prices corresponding to step (5) in the algorithm, and afterwards retrieve the stationary time series $Y(t)$ from the spot prices as in step (6). The plot on the right in Fig. 1 shows the filtered factor $X(t)$ along with the deseasonalized spot prices. It seems to reflect a long-term stochastic trend in the price data.

Next we estimate the mean reversion parameter $\eta$. The autocorrelation function of the time series $Y(t)$ is plotted in Fig. 2. Re-estimating $\eta$ over the first five lags results in $\widehat{\eta}=0.359$. The initial decrease of the autocorrelation function seems to be captured well by using an exponential function. However, it decays too rapidly for larger lags. Including more lags to fit the autocorrelation function (that is, $\eta$ ) results in a poor fit in the first lags. To get a better fit over all lags, one could use two (or more) exponential components. This would mean that we model the factor $Y$ by two or more Ornstein-Uhlenbeck processes, or by a higher-order CARMA model. Benth et al. [4] indicate that one should indeed use a higher-order CARMA model. However, such models are much more complex to estimate, and we apply the one-factor assumption on $Y$ here as a first approximation of the dynamics.

The next step is to fit a bivariate Lévy process $L=\left(L_{1}, L_{2}\right)$ to the time series $X(t)$ and $Y(t)$. For simplicity, we assume that $L_{1}$ and $L_{2}$ are independent, meaning that there is no dependency between the short-term and long-term price fluctuations. In the Schwartz-Smith model (see Schwartz and Smith [15], or Lucia and Schwartz [11] for the case of electricity) $L$ is assumed to be a bivariate Brownian motion. 


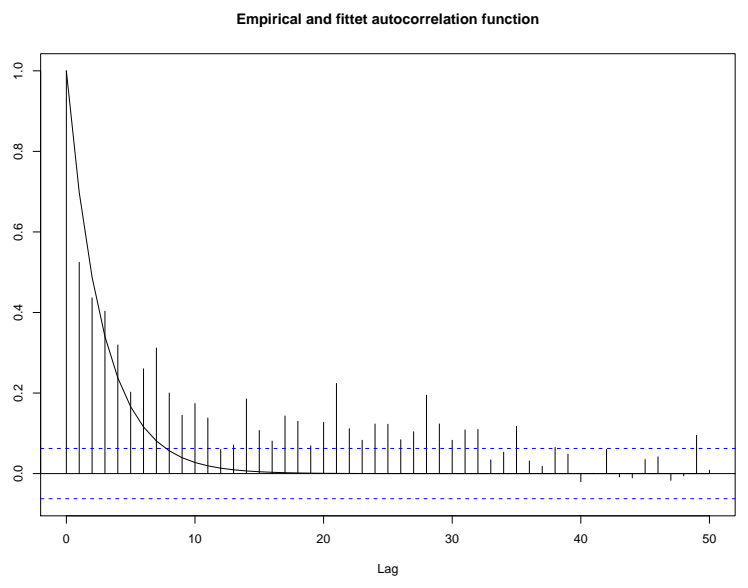

FIGURE 2. Autocorrelation function of $Y(t)$.
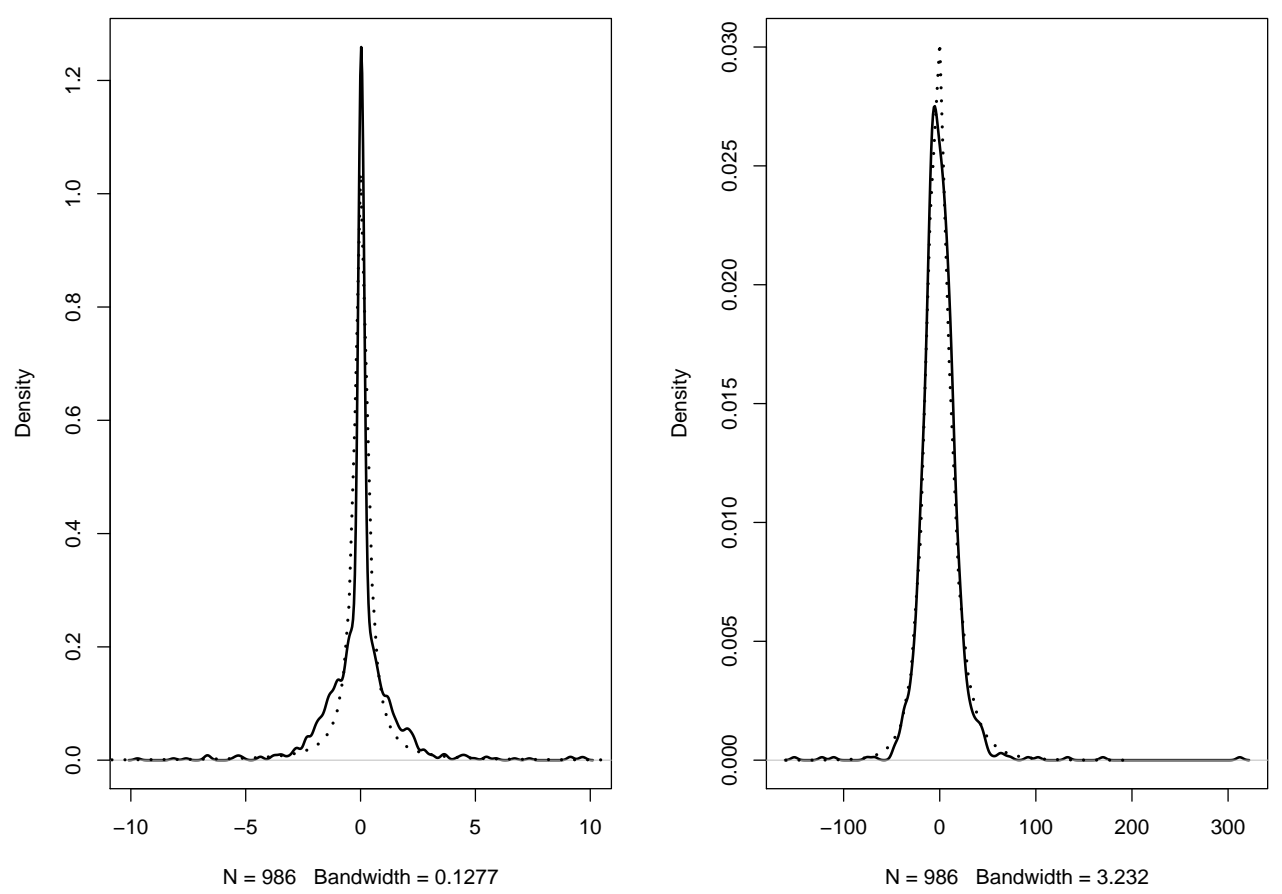

FIGURE 3. Empirical density of $L_{1}$ (left) and $L_{2}$ (right) as well as the fitted NIG density (dashed line).

However, the Gaussian assumption on the increments $\Delta X(t)$ is not realistic, and we propose to fit the dynamics of $X$ with a normal inverse Gaussian (NIG) Lévy process, that is, a Lévy process with NIG distributed marginals. The NIG distribution seems to be a good choice for modelling the residuals of $Y(t)$ as well.

The NIG distribution is a four parameter family of distributions successfully applied to model the logreturns of financial data. For its applications to finance and a detailed probabilistic analysis of the NIG family, we refer the interested reader to Barndorff-Nielsen [1]. Assuming $L_{1}(t)$ to be a NIG Lévy process, 


\begin{tabular}{ccccc}
\hline & $\alpha$ & $\beta$ & $\delta$ & $\mu$ \\
\hline$L_{1}$ & 0.0946 & -0.0099 & 0.3136 & 0.02421 \\
$L_{2}$ & 0.0402 & 0.0071 & 14.3407 & -2.9488 \\
\hline
\end{tabular}

TABLE 2. Estimated NIG parameters of $L_{1}$ and $L_{2}$.

\begin{tabular}{cccc}
\hline$i$ & $\theta_{i}$ & $\mathbb{E}_{P}\left[L_{i}(1)\right]$ & $\mathbb{E}_{\theta}\left[L_{i}(1)\right]$ \\
\hline 1 & 0.0115 & -0.0087 & 0.0296 \\
2 & 0.0010 & -0.3583 & 0.0211 \\
\hline
\end{tabular}

TABLE 3. The market price of risk derived from the fitted NIG parameters together with the expectation of $L_{1}$ and $L_{2}$ under $P$ and $Q_{\theta}$.

its cumulant (i.e., the logarithm of the characteristic function) function at time 1 is given by

$$
\Psi(x)=\delta\left\{\sqrt{\alpha^{2}-\beta^{2}}-\sqrt{\alpha^{2}-(\beta+i x)^{2}}\right\}+\mu i x,
$$

for the four parameters $\mu, \beta, \delta>0$ and $\alpha>0$. The skewness of the NIG distribution is described by $\beta$, where $\beta>0$ means a positively skewed distribution, and $\beta<0$ negatively skewed. For a symmetric NIG distribution, 1.e., when $\beta=0, \mu$ is the mean. Otherwise, $\mu$ is the location parameter. $\delta$ is the scale and $\alpha$ the tail heaviness parameter. Note that the NIG distribution has semi-heavy tails, with the normal distribution as a limiting case. We easily find the expectation from (4.2) as

$$
\kappa_{1}=\frac{\delta \beta}{\sqrt{\alpha^{2}-\beta^{2}}}+\mu \text {. }
$$

The estimated parameters of $L_{1}(1)$ based on maximum-likelihood are given in Table 2. We remark in passing that the NIG distribution has been applied in studies of energy prices in Benth and Šaltytè-Benth [5] and Börger et al. [8].

We fit another NIG Lévy process $L_{2}$ to the residuals of $Y$. The estimates are reported in Table 2. The estimated densities of $L_{1}(1)$ and $L_{2}(1)$ are displayed together with the empirical ones in Fig. 3. The fit seems to be good, and we find the NIG distribution as a satisfactory choice for modelling $L_{1}$ and $L_{2}$. Recall that we assumed independence of $L_{1}$ and $L_{2}$. Empirically, the correlation between the data series for $L_{1}$ and $L_{2}$ is given by -0.16 . A more realistic model should take this into account, which requires an analysis of the dependency structure. We relegate this to future studies. From the estimates in Table 2 we observe that the NIG distributions for $L_{1}$ and $L_{2}$ are close to symmetric.

Following step (8), the market price of risk $\theta=\left(\theta_{1}, \theta_{2}\right)$ is given by

$$
\begin{aligned}
& \theta_{1}=\frac{\alpha_{1} \frac{\hat{a}-\mu}{\delta}}{\sqrt{\left(\frac{\hat{a}-\mu_{1}}{\delta_{1}}\right)^{2}+1}}-\beta_{1} \\
& \theta_{2}=\frac{\alpha_{2} K}{\sqrt{K^{2}+1}}-\beta_{2},
\end{aligned}
$$

where

$$
K=\frac{\beta_{2}}{\delta_{2}}\left(\hat{c}-\frac{1}{2} \hat{a}\left(T_{2}-T_{1}\right)-\frac{\mu_{2}}{\beta_{2}}\right) .
$$

Here, the subscript in the parameters $\alpha, \beta, \delta$ and $\mu$ refer back to $L_{1}$ and $L_{2}$. Using the estimates for the NIG distributions, we can derive the values of $\theta_{1}$ and $\theta_{2}$. These are reported in Table 3 along with the expected values of $L_{1}$ and $L_{2}$ with respect to the probabilities $P$ and the fitted $Q_{\theta}$. We note that the market price of risk is positive, and that the expected value of $L_{1}$ and $L_{2}$ are moved from being negative under $P$ to positive under $Q_{\theta}$. The fitted market price of risk is shifting the distribution of $L_{1}$ and $L_{2}$ towards the right, roughly meaning that we get more positive jumps and less negative. Furthermore, quite nicely the NIG distribution is preserved under a constant Esscher transform. Hence, $L$ is a bivariate NIG Lévy process both under $P$ and $Q_{\theta}$, where only the skewness parameter is different under the two measures. 
Let us comment on the risk premium implied by our estimated model. The risk premium is defined as the difference between the futures price and the predicted average spot price over the delivery period. In mathematical terms,

$$
R_{P}\left(t, T_{1}, T_{2}\right)=F\left(t, T_{1}, T_{2}\right)-\mathbb{E}\left[\frac{1}{T_{2}-T_{1}} \int_{T_{1}}^{T_{2}} S(t) d t \mid \mathcal{F}_{t}\right] .
$$

From Proposition 3.2 we find

$$
\begin{aligned}
R_{P}\left(t, T_{1}, T_{2}\right)=\frac{1}{2} & \left(\mathbb{E}_{\theta}\left[L_{1}(1)\right]-\mathbb{E}\left[L_{1}(1)\right]\right)\left(T_{2}-T_{1}\right) \\
& +\left(\mathbb{E}_{\theta}\left[L_{1}(1)\right]-\mathbb{E}\left[L_{1}(1)\right]\right)\left(T_{1}-t\right) \\
& +\left(\mathbb{E}_{\theta}\left[L_{2}(1)\right]-\mathbb{E}\left[L_{2}(1)\right]\right) \frac{1}{\eta}\left(1-\bar{\eta}\left(t, T_{1}, T_{2}\right)\right) .
\end{aligned}
$$

The non-stationary factor gives a linear contribution in time to delivery $T_{1}-t$, while the stationary factor gives an exponential shape and converges fastly to a constant when $T_{1}-t \rightarrow \infty$. A plot of the risk premium for the estimated model parameters is shown in Fig. 4. As a result of the positive market price of risk, the

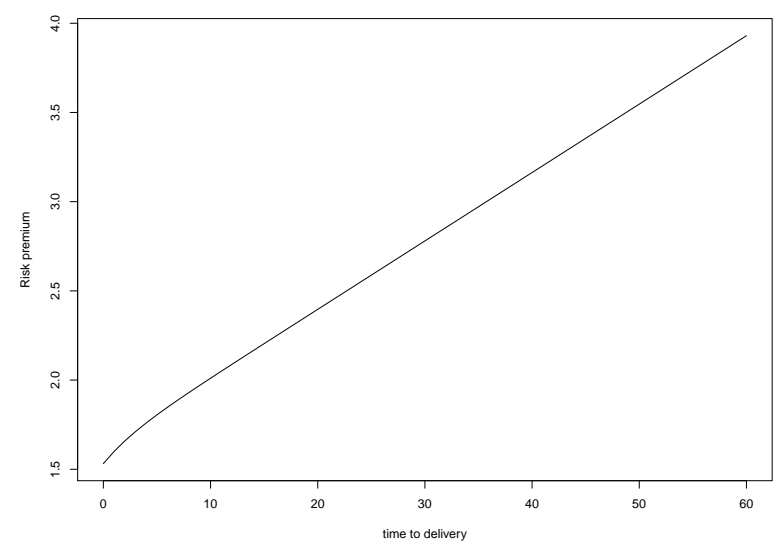

FIGURE 4. Theoretical risk premium for the estimated model parameters.

risk premium also becomes positive. This tells us that the consumers in the market are willing to pay a premium for locking in electricity prices in the futures market. Note that we use data from the relative short end of the market, using the front-month (or second month) contracts.

\section{PRICING OF OPTIONS ON FUTURES}

At EEX, the market for options is rather illiquid, however, there exists traded contracts. In 2008,12 options on baseload futures with delivery period 1 month were traded, 11 of them in the period we consider. Out of these 11, four are call options, and seven puts. We use these for further analysis and discussion.

In Tables 4 and 5 we list the calls and puts with their main characteristics. We have decided to label the contracts by $\mathrm{C} i, i=1,2,3,4$ for the calls and $\mathrm{P} i, i=1, \ldots, 7$ for the puts. Recall that the exercise time $\tau$ of the options is four trading days before the delivery period of the underlying futures starts. The historical data available from the EEX provides settlement prices for traded option contracts. For all derivatives traded, a settlement price is established on all exchange trading days. In the case that a settlement price cannot be determined on basis of the order book situation, a so-called Chief Trader Procedure applies, where all trading participants can take part with a representative. The EEX Market Supervision makes a standardised form available for all those trading participant volunteering to specify a market price for the respective derivatives. The settlement price is then determined as the average of the expectations of the market participants. We note that options on peakload futures are not traded at all at EEX, explaining why we use baseload spot data in our empirical analysis above. 


\begin{tabular}{cccccc}
\hline Contract & Trading day & Delivery period & Strike & Futures price & Settlement price \\
\hline C1 & 06.02 .2008 & Mar 2008 & 57 & 56.81 & 1.900 \\
C2 & 28.01 .2008 & Mar 2008 & 57 & 57.00 & 2.270 \\
C3 & 15.01 .2008 & Feb 2008 & 75 & 70.50 & 1.065 \\
C4 & 09.01 .2008 & Feb 2008 & 74 & 68.50 & 0.928 \\
\hline
\end{tabular}

TABLE 4. Traded call options in 2008 with delivery period 1 month.

\begin{tabular}{cccccc}
\hline Contract & Trading day & Delivery period & Strike & Futures price & Settlement price \\
\hline P1 & 08.07 .2008 & Aug 2008 & 74 & 74.77 & 3.233 \\
P2 & 08.07 .2008 & Aug 2008 & 75 & 74.77 & 3.835 \\
P3 & 03.07 .2008 & Aug 2008 & 73 & 78.00 & 1.989 \\
P4 & 08.04 .2008 & May 2008 & 55 & 55.35 & 1.522 \\
P5 & 04.03 .2008 & Apr 2008 & 58 & 58.70 & 1.911 \\
P6 & 28.02 .2008 & Apr 2008 & 58 & 61.75 & 0.955 \\
P7 & 08.01 .2008 & Feb 2008 & 65 & 69.00 & 1.179 \\
\hline
\end{tabular}

TABLE 5. Traded put options in 2008 with delivery period 1 month.

We first look at the "classical" approach to price options on futures in commodity markets, namely pricing using the Black-76 formula. For the convenience of the reader, we state the Black-76 formula in a Proposition.

Proposition 5.1. Suppose the risk-neutral futures price dynamics is a geometric Brownian motion

$$
\frac{d F\left(t, T_{1}, T_{2}\right)}{F\left(t, T_{1}, T_{2}\right)}=\sigma d B(t)
$$

for a constant $\sigma>0$. Then, the price at time $t \leq \tau$ of a call option with strike $K$ and exercise time $t \leq \tau \leq T_{1}$, is given by $C_{B 76}\left(t, F\left(t, T_{1}, T_{2}\right)\right)$ with

$$
C_{B 76}(t, x)=e^{-r(\tau-t)}\left[x \Phi\left(d_{1}(x)\right)-K \Phi\left(d_{2}(x)\right)\right],
$$

for $\Phi$ being the cumulative standard normal distribution function, and

$$
\begin{aligned}
& d_{1}(x)=\frac{\ln \left(\frac{x}{K}\right)+\frac{1}{2} \sigma^{2}(\tau-t)}{\sigma \sqrt{\tau-t}}, \\
& d_{2}(x)=d_{1}-\sigma \sqrt{\tau-t} .
\end{aligned}
$$

In the Black-76 formula, one boldly assumes the futures price dynamics to be a geometric Brownian motion, a dynamics which is far from the one we have estimated to the electricity futures prices at the EEX. The volatility $\sigma$ is also constant, an assumption that is not likely to be true. Based on the historically estimated volatility of the futures contracts in question, we can price the call options. The Black-76 prices are reported in Table 6 along with the actual settlement prices as quoted on the EEX. Appealing to the putcall parity, we report the put prices in Table 7. In both tables, we have also reported the historical volatility $\sigma$ used in the Black-76 formula, as well as the implied volatility so that Black-76 matches the settlement price. We estimate the historical volatility of the logreturns of the underlying futures from the last month of daily price data. Furthermore, we choose $r=5 \%$ which is about the average yearly Euro LIBOR rate in 2008. We find that the price of all options are substantially underestimated by Black-76. Due to the low volatility, those options that are far out of the money have a Black-76 price being essentially 0 (P6 and P7, and C3 and C4). The implied volatility becomes very high compared to the historical volatility. Indeed, the historical volatility is in the modest range of $8-11 \%$ for the underlying futures of the call, whereas the implied volatilities are estimated to be from $35 \%$ to $50 \%$. The mispricing is rather dramatic, as the percentages ranging above $70 \%$ tells. One might be tempted to speculate that the market is adding a huge risk premium for effects like illiquidity of the options and non-normality of the futures price dynamics. 


\begin{tabular}{cccccc}
\hline Contract & Settlement price & Black-76 & Mispricing & hist. vol. & impl. vol. \\
\hline C1 & 1.900 & 0.464 & $-76 \%$ & 0.1046 & 0.3770 \\
C2 & 2.270 & 0.725 & $-68 \%$ & 0.1100 & 0.3560 \\
C3 & 1.065 & 0.000 & $-100 \%$ & 0.0788 & 0.5030 \\
C4 & 0.928 & 0.000 & $-100 \%$ & 0.0821 & 0.4450 \\
\hline
\end{tabular}

TABLE 6. Black-76 pricing of the call options

\begin{tabular}{cccccc}
\hline Contract & Settlement price & Black-76 & Mispricing & hist. vol. & impl. vol \\
\hline P1 & 3.233 & 0.693 & $-79 \%$ & 0.1491 & 0.521 \\
P2 & 3.835 & 1.158 & $-70 \%$ & 0.1491 & 0.532 \\
P3 & 1.989 & 0.055 & $-97 \%$ & 0.1496 & 0.509 \\
P4 & 1.522 & 0.177 & $-88 \%$ & 0.0679 & 0.357 \\
P5 & 1.911 & 0.295 & $-85 \%$ & 0.1014 & 0.394 \\
P6 & 0.955 & 0.001 & $-100 \%$ & 0.0797 & 0.366 \\
P7 & 1.179 & 0.000 & $-100 \%$ & 0.0842 & 0.437 \\
\hline
\end{tabular}

TABLE 7. Black-76 pricing of the put options

The issuer runs a big risk selling call options, since it is difficult to turn around the position in the option market. However, the underlying futures is reasonably liquid, so delta hedging is possible. This removes some of the liquidity risk for the issuer.

One can in theory create synthetic investment strategies mimicking to a large extent the payoff of a call or put option. This could be used in order to exploit potential arbitrages in the option market. However, if the futures dynamics is not a geometric Brownian motion, there will be a large residual error in such strategies, which theoretically can be made perfect by delta hedging in the Black-76 framework. The empirical study of spot and futures pricing in the previous Section strongly points towards non-Gaussian models, hence ruling out this possibility.

In any case, the conclusion so far is that Black-76 in its simplest form is inadequate for pricing of options in the EEX market. As our proposed futures price dynamics is far more sophisticated than a simple geometric Brownian motion, we now move on to analyse the implied option prices from this model with the hope that it can improve the situation.

The call option price is then given by

$$
C(t)=e^{-r(\tau-t)} \mathbb{E}_{Q}\left[F\left(\tau, T_{1}, T_{2}\right)-K \mid \mathcal{F}_{t}\right] .
$$

The pricing probability $Q$ is an equivalent martingale measure for $F\left(t, T_{1}, T_{2}\right)$, and we let this be given by $Q_{\theta}$. The $Q_{\theta}$-dynamics of $F\left(t, T_{1}, T_{2}\right)$ is given by Proposition 3.3 and $Q_{\theta}$ is determined through the market price of risk (4.3) from the spot-futures analysis above. We evaluate the expectation through a MonteCarlo simulation. To simulate the Lévy processes $L_{1}$ and $L_{2}$ under $Q_{\theta}$, we use that NIG-Lévy processes are stable with respect to an Esscher change of measure. In fact, it can be seen (see Benth et al. [3]) that if, for $i=1,2, L_{i}(1)$ is NIG distributed under $P$ with parameters $\alpha_{i}, \beta_{i}, \delta_{i}$ and $\mu_{i}$, then the $L_{i}(1)$ is again NIG distributed under $Q_{\theta}$ with the same parameters except the skewness, which becomes $\beta_{i}+\theta_{i}$.

Based on a simulation of 1,000,000 paths we compute the option prices based on the average payoff. To simulate the NIG distribution, we applied the algorithm implemented in the R-package fBasics, which is based on the normal variance-mean mixture of the NIG distribution.

The resulting numbers are reported in Tables 8 and 9. We have also included the mispricing and computed the implied volatility of the simulated price using the Black-76. From the tables, we see that the picture is more mixed, with both over and underpricing of the calls and puts. Moreover, at the first glance, the mispricing seems to be less severe than in the case of Black-76, although admittedly still very big.

Our spot and futures price model includes non-Gaussian noise as both factors in the spot are driven by an NIG Lévy process. Note that the futures price is depending on the non-stationary factor directly, whereas the short-term factor is dampened and negligible for contracts far from delivery. From our estimation 


\begin{tabular}{ccccc}
\hline Contract & Settlement price & Simulated price & Mispricing & impl. vol. \\
\hline C1 & 1.900 & 2.748 & $45 \%$ & 0.4820 \\
C2 & 2.270 & 3.525 & $56 \%$ & 0.4882 \\
C3 & 1.065 & 0.821 & $-23 \%$ & 0.4255 \\
C4 & 0.928 & 1.006 & $9 \%$ & 0.4037 \\
\hline
\end{tabular}

TABLE 8. Simulated prices of the call options.

\begin{tabular}{ccccc}
\hline Contract & Settlement price & Simulated price & Mispricing & impl. vol. \\
\hline P1 & 3.233 & 2.476 & $-23 \%$ & 0.4256 \\
P2 & 3.835 & 2.964 & $-23 \%$ & 0.4239 \\
P3 & 1.989 & 1.438 & $-28 \%$ & 0.4260 \\
P4 & 1.522 & 2.397 & $57 \%$ & 0.5358 \\
P5 & 1.911 & 2.659 & $39 \%$ & 0.5368 \\
P6 & 0.955 & 1.889 & $98 \%$ & 0.5384 \\
P7 & 1.179 & 1.376 & $17 \%$ & 0.4032 \\
\hline
\end{tabular}

TABLE 9. Simulated prices of the traded put options.

procedure, the non-stationary long-term factor is estimated from the futures prices, so if the market would price according to our futures price dynamics with the given pricing measure $Q_{\theta}$, at least options with long time until exercise should be priced reasonably accurate. Looking at $\mathrm{C} 1$ and $\mathrm{C} 2$, these have the longest time to exercise in our sample of call options. However, the simulated option prices from our model for these two contracts are approximately $50 \%$ higher than the quoted prices. This means that our model is pricing in too much risk. From the spot dynamics we estimated positive market prices of risk which pushes the skewness of the NIG distribution to more positive jumps. The more positive market price of risk, the higher values of the options. Thus, it seems like the futures model inherits far too much risk premium from the spot when it comes to option pricing. We reach the conclusion that the option market is not including the same risk perception as the one inherited from the spot in the futures market. This is a clear sign that a completely different pricing measure $Q$ is used in the option market than in the futures pricing. Note that $\mathrm{C} 1$ and $\mathrm{C} 2$ are both (approximately) at-the-money, so a big portion of the distribution of the futures is taken into account in the pricing.

The contracts C3 and C4 are far out-of-the-money and slightly closer to exercise time than C1 and C2. Noteworthy is that the mispricing of these are significantly less than for $\mathrm{C} 1$ and $\mathrm{C} 2$, being respectively $-23 \%$ and $9 \%$. If we have based our calculations of the call prices on the wrong risk premium, it will be more influential far from exercise than close since we span out more of the risk the longer into the future we simulate the futures price. Close to exercise, the misspecification of the tails under the chosen $Q$ will be relatively much smaller than when we move futures. Maybe more importantly is that a smaller portion of the price distribution of $F$ is taken into account for these two out-of-the-money options than $\mathrm{C} 1$ and $\mathrm{C} 2$, and hence a wrongly chosen $Q$ matters less. This discussion conforms with the observations above for $\mathrm{C} 1$ and $\mathrm{C} 2$.

Note that contract P6 is farthest from exercise among the put options, as well as being out-of-the-money. This contract has the highest mispricing by our model. All the other put contracts in our sample have shorter time left to exercise. P1, P2, P4 and P5 are all approximately at-the-money put options with almost the same time left until exercise. The mispricing of these are significantly less than for P6. In fact, for P1 and P2 our model gives a price $-23 \%$ less than the settlement prices. P4 and P5 are contracts on futures with delivery in the spring months May and April, respectively. Temperature predictions may influence the futures price expectations, as the spring may become colder or warmer than usual. We also note that it is the left-tail of the futures price distribution that counts when pricing an out-of-the-money put option. An underpricing can be the result of the distribution being moved to the right by a positive risk premium. 
P3 and P7 are out-of-the-money put options where the mispricing of our model is rather modest $(-28 \%$ and $17 \%$, respectively). P7 is the only put option written on a futures with delivery in the winter period, namely February. For the calls $\mathrm{C} 3$ and C4, which also are written on February futures contacts, we observe a relatively small pricing error. It seems like the model captures best the futures price evolution in the winter term. We also remark that the poor fit of the autocorrelation of the stationary factor $Y$ may lead to wrong assessments of the spike influence. However, we believe that this is to some extent compensated for by the good fit of the Lévy process $L_{2}$ using a NIG distribution.

All in all, it seems like the futures price dynamics based on the pricing measure $Q_{\theta}$ implied by the spotfutures relationship provides a significantly better prediction of option prices than Black-76. However, the prices are far from satisfactory, and we find clear evidence that the risk-adjustments should be different than those given by $Q_{\theta}$. Based on our findings, we dare to conclude that another pricing measure $\widetilde{Q}$ should be used for power option pricing, a pricing measure which attributes a different loading on the distributions of the Lévy processes $L_{1}$ and $L_{2}$. In fact, based on the differences between summer and winter contracts in the pricing analysis above, one may suspect that such a measure change should incorporate seasonalities as well. Furthermore, it may also account for the state of the futures price, so that one can capture outof and in-the-money option price differences better. One can also think of pricing measures which not only changes the characteristics of the jump processes $L_{1}$ and $L_{2}$, but as well change the dynamics. For example, it is possible to define measures which change the speed of mean reversion of the $Y$ factor. This could for example lead to a slower risk-neutral speed of mean reversion, essentially saying that a spike lasts longer in a risk neutral context than under the market probability.

As our futures price dynamics consists of two jump components, it gives rise to an incomplete market model. The selection of risk neutral probabilities for pricing options in such markets is frequently based on utility indifference pricing techniques (see Rouge and El Karoui [12]). Such a method, which is based on a risk averse, utility optimizing investor, leads to a partial hedging strategy of the option. The utility indifference method is particularly useful when pricing options in illiquid markets, where one is stuck with the option investment. Other approaches to pricing is based on deriving optimal partial hedges, where the optimality criterion may be the futures investment hedge which minimizes the variance of the hedging error (see Cont and Tankov [9]). All these various approaches lead to a pricing measure $Q$. It is of great interest and application to see whether such prices will explain the settlement option prices in the EEX market, and whether our conjecture $\widehat{Q} \neq \widetilde{Q}$ is true.

As we have mentioned earlier, the option market at the EEX is rather illiquid, and a liquidity premium is naturally associated to the observed prices. This premium will be part of the risk premium as we have estimated. Once bought or sold an option, one might be stuck with the position taken until exercise or having to accept a significant loss by reversing it. This will impact the settlement prices as buyers and sellers know that it is difficult to get out of the position at a later time. On the other hand, the underlying futures market is reasonably liquid, so any position can (in theory, at least) be hedged to a certain degree. Considering our derived Black-76 prices, which were consistently too high, one could speculate whether the sellers had to accept a discount in prices due to illiquidity. However, for the much more realistic futures price dynamics that we considered, the picture was more mixed with both over- and underpricing. There is no doubt that a liquidity premium exists in the market, but it is hard from our analysis to conclude anything on its size and structure. Moreover, liquidity might also be an issue in the futures market, further complicating matters.

\section{CONCLUSION}

We have argued that in power markets one may use a probability measure $\widehat{Q}$ for futures pricing based on spot modelling which can be different than the equivalent martingale measure $\widetilde{Q}$ used for pricing options on the futures. There is no violation of no-arbitrage pricing theory that $\widehat{Q} \neq \widetilde{Q}$, and the argument hinges on the fact that electricity spot cannot be stored. Due to the non-storability, $\widetilde{Q}$ can be chosen as an equivalent measure which is not necessarily turning the discounted spot dynamics into a $\widetilde{Q}$-martingale. On the other hand, $\widehat{Q}$ is an equivalent measure such that the futures price becomes a $\widehat{Q}$-martingale.

We introduce a two-factor model for the spot price dynamics being a generalization of the classical commodity model of Schwartz and Smith [15]. Both the long-term and the short-term factors are driven by normal inverse Gaussian Lévy processes, a choice based on empirical arguments using data collected 
at the EEX. The spot model allows for analytical futures pricing, where the Esscher transform provides an parametric class of probability measures to model the risk premium. We perform a joint estimation of the spot and futures, where the crucial step is to apply long-dated futures contracts to filter out the non-stationary long-term factor of the spot.

Applying Monte Carlo simulations we priced call and put option prices for our proposed futures dynamics. We compared the simulated prices where we chose $\widehat{Q}=\widetilde{Q}$ with observed option prices in the market. This led to a significant mispricing, and we argued that the results pointed to the fact that $\widetilde{Q} \neq \widehat{Q}$. Our results were benchmarked against the Black-76 prices using the historical volatility of the underlying futures as input. The proposed spot-futures model was a clear improvement over this in predicting option prices.

We did not suggest any probability $\widehat{Q}$ for the futures price which could remedy the situation. There exists many potential approaches to produce such risk neutral probabilities taken from the theory of derivatives pricing in incomplete markets. But before setting off such a study, one should make the spot dynamics even more sophisticated to take into account some defiance like the misspecification of the autocorrelation structure of the stationary factor. CARMA processes could be a choice here, or even more general Lévy semistationary processes. However, this will require more advanced estimation procedures to fit to data. On the other hand, such improvements will make the conclusions on option pricing and choice of risk neutral measures less prone to model error. A further issue is to open for more flexible pricing measures for the futures price, taking into account random changes in the risk premium and impacts from fuels and weather.

Illiquidity of the power option markets is a clear issue which can question our analysis. Power options are relatively little traded, but we believe that in the future these markets will emerge as important one for hedging and speculation of power. The results in our paper will hopefully provide an important guideline in the challenges when pricing spot, futures and options simultaneously.

\section{REFERENCES}

[1] Barndorff-Nielsen OE (1998) Processes of normal inverse Gaussian type. Finance Stoch 2(1):41-68.

[2] Barndorff-Nielsen OE, Benth FE, Veraat AED (2013) Modelling energy spot prices by volatility modulated Lévy-driven Volterra processes. Bernoulli 19(3):803-845.

[3] Benth FE, Šaltytė-Benth J, Koekebakker S (2008) Stochastic Modelling of Electricity and Related Markets. World Scientific, Singapore

[4] Benth FE, Klüppelberg C, Müller G, Vos L (2011) Futures pricing in electricity markets based on stable CARMA spot models. Preprint.

[5] Benth FE, Šaltytė-Benth J (2004) The normal inverse Gaussian distribution and spot price modelling in energy markets. Int J Theor Appl Finance 7(2):177-192.

[6] Benth FE, Sgarra C (2012) The risk premium and the Esscher transform in power markets. Stoch Analysis Appl 30:20-43.

[7] Black F (1976) The pricing of commodity contracts. J Financial Econ 3:169-179.

[8] Börger R, Cartea A, Kiesel R, Schindlmayer G (2009) A multivariate commodity analysis and applications to risk management. J Futures Mark 29(3):197-217.

[9] Cont R, Tankov P (2004) Financial Modelling with Jump Processes. Chapman \& Hall/CRC, Boca Raton

[10] Duffie D (1992). Dynamic Asset Pricing Theory. Princeton University Press, Princeton

[11] Lucia JJ, Schwartz ES (2002) Electricity prices and power derivatives: evidence from the Nordic Power Exchange. Rev Deriv Res 5(1):5-50.

[12] Rouge R, El Karoui N (2000) Pricing via utility maximization and entropy. Math Finance 10:259-276.

[13] Samuelson P (1965) Proof that properly anticipated prices fluctuate randomly. Indust Manag Rev 6:41-44.

[14] Shiryaev AN (1999) Essentials of Stochastic Finance. World Scientific, Singapore

[15] Schwartz E, Smith JE (2000) Short-term variations and long-term dynamics in commodity prices. Manag Science 46(7):893-911.

(Fred Espen Benth), Centre of Mathematics for ApPlications, University of Oslo, P.O. Box 1053, Blindern, N-0316 OSLO, NORWAY

E-mail address: fredbemath.uio.no

URL: http://folk.uio.no/fredb/

(Maren Diane Schmeck) Department of MATHEMATICs, University of Cologne, Weyertal 86-90, 50931 Cologne, GERMANY

E-mail address: mschmeck@math. uni-koeln.de

URL: http://www.mi.uni-koeln.de/ mschmeck/ 